\title{
Modified radical mastectomy for level III axillary lymph node clearance: a case report
}

\author{
Mingkun Zhang, Liu Yang, Lan Hou, Zhe Wang, Juliang Zhang \\ Department of Thyroid, Breast, and Vascular Surgery, Xijing Hospital, The Fourth Military Medical University, Xi'an, China \\ Correspondence to: Juliang Zhang. Department of Thyroid, Breast, and Vascular Surgery, Xijing Hospital, The Fourth Military Medical University, \\ 127 Changle West Road, Xi'an 710032, China. Email: vascularzhang@163.com.
}

\begin{abstract}
While modified radical mastectomy with level I and level II axillary lymph node clearance is a typical operating method in breast surgery, level III axillary lymph node clearance is necessary in some cases such as those involving apical axillary nodes. Level III dissection can provide accurate postoperative staging and essential guidance for postoperative adjuvant therapy. Although it is often difficult to expose the subclavian region and dissect level III axillary lymph nodes, in this case, the author split the pectoralis major muscle $2 \mathrm{~cm}$ inferior to the collarbone and performed a skeletonized complete level III axillary lymph node dissection. The author cut apart the fat on the surface of subclavian vein, lifted the fascia on the surface of the subclavian vein, removed the lymphoid adipose tissue along the fascial space completely and skeletonized subclavian vein. This approach provides less operating space, but it can fully expose the subclavian area, making it easier to dissociate and dissect the parasternal ligament, subclavian vein, medial border of the pectoralis minor muscle, and other important anatomical landmarks. In addition, the pectoralis branches of the thoracoacromial artery and the lateral cutaneous branches of the intercostal nerves were protected when removing the axillary nodes, which reduced postoperative complications such as upper limb numbness, tingling sensation, and muscle atrophy. Axillary lymph nodes were completely resected from inside to outside, and the important anatomical markers of axilla such as axillary vein, long thoracic nerve, thoracodorsal nerve and thoracodorsal vessels were clearly exposed.
\end{abstract}

Keywords: Breast cancer; level III axillary lymph nodes clearance; branches of thoracoacromial artery; lateral cutaneous branches of intercostal nerves; case report

Submitted Jul 13, 2021. Accepted for publication Sep 09, 2021.

doi: $10.21037 / g s-21-567$

View this article at: https://dx.doi.org/10.21037/gs-21-567

\section{Introduction}

Breast carcinoma is the most common malignant tumor in females and the second most common cause of cancer-related mortality in females (1). Axillary node clearance is still the cardinal operation method in patients receiving breast mastectomy surgery with positive sentinel lymph nodes and in patients receiving breast conservation treatment with three or more sentinel lymph node metastasis, although axillary management in breast cancer is still controversial (2). Axillary lymph node dissection is of great importance to evaluate the prognosis and develop treatment plans for breast cancer patients. The surgical approach presented in this paper can make it easier to dissect axillary lymph nodes and reduce the risk of nerve or vessel injury during dissection.

Usually, the pectoralis minor is an essential anatomical marker which divides axillary lymph nodes into three levels. Metastasis of level III axillary lymph nodes means poor prognosis. The National Comprehensive Cancer Network (NCCN) guidelines for breast cancer demonstrate that in the absence of gross disease in level II nodes, lymph node dissection should include tissue inferior to the axillary vein from the latissimus dorsi muscle laterally to the medial border of the pectoralis minor muscle (level I/II) (3), while level III dissection to the thoracic inlet should be 
performed only in cases with gross disease in level II and/ or III (4). It is often difficult to expose the subclavian region and dissect level III axillary lymph nodes. Muscolino et al. proposed a practical method to reach level III axillary lymph nodes: splitting the pectoralis major muscle and immobilizing the pectoralis minor muscle (5). The research of Hadjiminas et al. showed that access to level III through a muscle splitting transverse incision on the pectoralis major provided excellent visualization of level III (6). In the present operation, the surgeon performed an elaborative method to protect the important structures of the armpit, and completed a thorough skeletonized dissection of level I, II, and III axillary lymph nodes. We present the following article in accordance with the CARE reporting checklist (available at https://dx.doi.org/10.21037/gs-21-567).

\section{Case presentation}

A 56-year-old female with no personal or family history of cancer presented to the outpatient department with the primary complaint of discovering a lump on the right breast 3 months previous. On breast examination, there was a hard mass of about $3 \mathrm{~cm}$ diameter and with good mobility located in the inner upper quadrant of the right breast, and an enlarged lymph node was present in the right axilla. At color doppler ultrasound, a hypoechoic mass measuring approximately $3.2 \mathrm{~cm} \times 2.5 \mathrm{~cm}$ was visible at the inner upper quadrant of the right breast (BIRADS 5). Swollen lymph nodes with abnormal structure were seen in the right axillary and subclavian region, and the boundary between the cortex and medulla of the nodes was unclear. At Molybdenum target, a hi-density calcification area with indistinct margins was observed in the right breast (BIRADS $4 C)$. No distant metastasis was found in the baseline assessment.

Ultrasound-guided core needle biopsy showed breast invasive carcinoma (low-grade). Immunohistochemistry (IHC) revealed estrogen receptor (ER), progesterone receptor (PR), and human epidermal growth factor receptor 2 (Her2) were negative on the tumor cells, and the Ki-67 index was $50 \%$. Fine needle biopsy revealed cancer cells in the right axillary and right subclavian lymph nodes. The preoperative diagnosis was right breast cancer (cT2N3M0, stage IIIc), and before surgery, the patient received chemotherapy $(\mathrm{EC} \times 4 \rightarrow \mathrm{T} \times 4)$. After neoadjuvant chemotherapy, the patient received a modified radical mastectomy, and the postoperative pathology showed fibrostromal hyperplasia of the breast with hyaline degeneration and local foam cell aggregation, which conformed to the changes after chemotherapy. In the axillary lymph nodes, one of the fourteen level I nodes was involved (1/14); no cancer metastasis was found in four level II nodes (0/4); one of the three level III nodes was involved (1/3); and there was no metastasis in the one intermuscular lymph node $(0 / 1)$. The postoperative diagnosis was right breast cancer (pTxN3M0, stage IIIc). After surgery, the patient underwent standard-dose radiotherapy which following the statement of National Cancer Institute (7), and based on the result of CREATE-X research, we recommended her for adjuvant treatment with capecitabine (8). All procedures performed in studies involving human participants were in accordance with the ethical standards of the institutional and/or national research committee(s) and with the Helsinki Declaration (as revised in 2013). Written informed consent was obtained from the patient for publication of this case report and accompanying images. A copy of the written consent is available for review by the editorial office of this journal.

\section{Surgical procedure}

We presented the procedure of the operation in detail (Video 1). A fusiform incision was performed around the nipple and areola, the skin on the surface of the tumor, and the puncture needles pinhole on the right breast. The epidermis was slit with a scalpel, and the dermis and subcutaneous tissue incised with an electro tome. The assistant nipped the dermis with mosquito forceps and pulled the skin flap upwards, and the operator pushed the breast in the opposite direction with gauze. The author then used electro tome to dissociate the flaps in the superficial layer of the superficial fascia of the breast, at the attachment point of the Cooper ligament. The operation region of the flaps accorded to the predefined range of the line drawn before operation, which saw the upper border two fingers below the clavicle, the lower border as the costal arch, the medial border as the side of the sternum, and the lateral border the anterior edge of the latissimus dorsi. The author lifted the superior margin of the breast by tissue forceps, then excided breast tissue and the deep layer of superficial fascia along the direction of pectoralis major fibers by electro tome to coagulate the perforator vessel when removing interior breast tissue. Electro tome was also used to cut apart the pectoralis major along the direction of muscle fibers, then fat on the surface of the pectoralis minor muscle was removed to reveal the muscle, while paying attention to protect the pectoralis branches of the thoracoacromial artery. Assistants then pulled the pectoralis major and minor muscles to both 
sides with hooks and exposed the subclavian area fully. The author then dissected level III axillary lymph nodes carefully at a range: up to the subclavian vein, down to the entrance of the thorax, inside to the parasternal ligament, and outside to the medial edge of pectoralis minor muscle. Level III lymph nodes were marked with a suture knot.

The pectoralis major and pectoralis minor muscle were separated along their interval, and intermuscular lymph nodes were resected. The coracoclavicular fascia along the lateral border of the pectoralis minor muscle was opened and the armpit entered, while attention was paid to protect the lateral thoracic artery and vein. The tongue-like fat was then cut apart to expose the axillary vein, and the long thoracic nerve was dissociated and exposed along the lateral margin of the serratus anterior muscle. One assistant pulled the pectoralis minor to the inner side, and the surgeon then removed level II and level III axillary lymph nodes. The author then freed and protected three lateral cutaneous branches of the intercostal nerves including the intercostobrachial nerve, which is the second lateral cutaneous branch of intercostal nerve. Lastly, the author dissected level I axillary lymph nodes, which are divided into an inner triangle and outer triangle. The inner triangle area was then cleaned at the range: superior to the axillary vein, external to the thoracodorsal vessels, and internal to the long thoracic nerve, while paying attention to protect the long thoracic nerve, dorsal thoracic nerve, dorsal thoracic artery and vein, and their branches. The outer triangle area was cleaned at the range: superior to the axillary vein, internal to the thoracodorsal vessels, and external to the lateral margin of the latissimus dorsi muscle, while paying attention to protect the subscapular vessels, thoracodorsal nerves, thoracodorsal arteries and veins, and their branches. The axillary lymph nodes and breast specimens were then completely removed. The operative cavity was checked carefully for bleeding, then flushed with sterile distilled water and normal saline, and two drainage tubes placed. The skin and subcutaneous tissue were then closed with inverting suture.

\section{Discussion}

Many scholars believe that level III axillary lymph node resection is an unnecessary and extreme procedure for the majority of breast carcinomas (9), as its benefits are uncertain, and it can lead to a series of postoperative complications such as paresthesia, armpit dysmorphia, and upper limb edema. In T1/T2/T3 and N0/N1a/N1b breast carcinoma patients, Kodama et al. contrasted the effectiveness of level III axillary lymph node resection and level I axillary lymph node resection, and the results indicated that the former was unable to improve prognosis (10). The research of Tominaga et al. showed that in patients receiving level II or level III axillary lymph node clearance, the 10-year overall survival (OS) rates were $86.6 \%$ and $85.7 \%(\mathrm{P}=0.931)$, respectively, and 10-year disease-free survival (DFS) rates were $73.3 \%$ and $77.8 \%(\mathrm{P}=0.666)$, respectively, indicating that level III axillary lymph node clearance was unnecessary for patients with stage II breast carcinoma (11).

Although level III dissection does not ameliorate survival, it can provide accurate postoperative staging and essential guidance for postoperative adjuvant therapy. Metastasis of level III axillary lymph nodes is an essential factor that causes local recurrence and distant metastasis. Level III dissection can screen out patients at high risk of recurrence for intensive therapy. In this case, level III axillary lymph nodes were dissected because of positive subclavian lymph nodes puncture. There are usually two approaches for level III axillary lymph node dissection, with some surgeons retracting the pectoralis major and minor muscles, while others prefer to split the pectoralis major muscle (Kodama method). When applying Kodama method, surgeons usually split the pectoralis major muscle through intermuscular sulcus. However, the position of intermuscular sulcus is often too high to access to subclavian region and intermuscular sulcus is absent in some patients. In this case, the authors split the pectoralis major muscle $2 \mathrm{~cm}$ below the clavicle along the direction of the pectoralis major fiber, which make access and visualization much easier. Although this approach provides less operating space, it can fully expose the subclavian area, making it easier to dissociate and dissect the parasternal ligament, subclavian vein, medial border of the pectoralis minor muscle, and other important anatomical landmarks. At the same time, due to the splitting of the pectoralis major, the dissection of lymph nodes between the pectoralis major and minor muscles is more thorough, and the protection of the pectoralis branch of the thoracoacromial artery will be easier. While cleaning the lymph nodes of the subclavian region, lymph fat tissue should be dissected towards the back of the pectoralis minor muscle as far as possible, which will make it easier to pull the level III lymph nodes out from the outside edge of the muscle.

The intercostal nerves send out lateral cutaneous branches near the anterior axillary line. The lateral cutaneous branch of the second intercostal nerve is distributed in 
the medial arm skin across the armpit, where it is called the intercostobrachial nerve. This nerve is an important anatomical structure of the axilla, closely related to the abnormal sensory function sometimes seen after operation. The intercostobrachial nerve usually originates in the second intercostal space, and often merges with the first and third intercostal nerve branches $(12,13)$. In the present operation, the surgeon carefully dissected and exposed the lateral cutaneous branches of the intercostal nerves and intercostobrachial nerve on the side of the chest wall, freeing them completely, while paying attention to keep the electro tome away from the nerves. While cleaning the internal triangle, attention was paid to protect the branches of the thoracic dorsal vessels, especially the branches of the serratus anterior muscle, which is easily injured when cleaning the side of chest wall. The circumflex scapular artery and vein should also be protected when cleaning the external triangle.

\section{Acknowledgments}

Funding: None.

\section{Footnote}

Reporting Checklist: The authors have completed the CARE reporting checklist. Available at https://dx.doi. org/10.21037/gs-21-567

Conflicts of Interest: All authors have completed the ICMJE uniform disclosure form (available at https://dx.doi. org/10.21037/gs-21-567). The authors have no conflicts of interest to declare.

Ethical Statement: The authors are accountable for all aspects of the work in ensuring that questions related to the accuracy or integrity of any part of the work are appropriately investigated and resolved. All procedures performed in studies involving human participants were in accordance with the ethical standards of the institutional and/or national research committee(s) and with the Helsinki Declaration (as revised in 2013). Written informed consent was obtained from the patient for publication of this case report and accompanying images. A copy of the written consent is available for review by the editorial office of this journal.

Open Access Statement: This is an Open Access article distributed in accordance with the Creative Commons
Attribution-NonCommercial-NoDerivs 4.0 International License (CC BY-NC-ND 4.0), which permits the noncommercial replication and distribution of the article with the strict proviso that no changes or edits are made and the original work is properly cited (including links to both the formal publication through the relevant DOI and the license). See: https://creativecommons.org/ licenses/by-nc-nd/4.0/.

\section{References}

1. Fahad Ullah M. Breast cancer: current perspectives on the disease status. Adv Exp Med Biol 2019;1152:51-64.

2. Veronesi P, Corso G. Standard and controversies in sentinel node in breast cancer patients. Breast 2019;48 Suppl 1:S53-6.

3. Gradishar WJ, Anderson BO, Balassanian R, et al. NCCN guidelines insights: breast cancer, version 1.2017. J Natl Compr Canc Netw 2017;15:433-51.

4. National Comprehensive Cancer Network. NCCN guidelines for the treatment of breast cancer. 2017. Available online: https://www.nccn.org/professionals/ physician_gls/pdf/breast.pdf

5. Muscolino G, Leo E, Sacchini V, et al. Resectable breast cancer: axillary dissection sparing pectoralis muscles and nerves. Eur J Surg Oncol 1988;14:429-33.

6. Hadjiminas DJ, Zacharioudakis KE. Direct transpectoral approach for level III axillary lymph node clearance. Ann R Coll Surg Engl 2014;96:481-2.

7. Buchholz TA, Lehman CD, Harris JR, et al. Statement of the science concerning locoregional treatments after preoperative chemotherapy for breast cancer: a National Cancer Institute conference. J Clin Oncol 2008;26:791-7.

8. Masuda N, Lee SJ, Ohtani S, et al. Adjuvant capecitabine for breast cancer after preoperative chemotherapy. $\mathrm{N}$ Engl J Med 2017;376:2147-59.

9. Kaufmann M, Morrow M, von Minckwitz G, et al. Locoregional treatment of primary breast cancer: consensus recommendations from an International Expert Panel. Cancer 2010;116:1184-91.

10. Kodama H, Nio Y, Iguchi C, et al. Ten-year follow-up results of a randomised controlled study comparing level-I vs level-III axillary lymph node dissection for primary breast cancer. Br J Cancer 2006;95:811-6.

11. Tominaga T, Takashima S, Danno M. Randomized clinical trial comparing level II and level III axillary node dissection in addition to mastectomy for breast cancer. $\mathrm{Br} \mathrm{J}$ Surg 2004;91:38-43. 
12. Zhu J, Jiao D, Guo X, et al. Predictive factors and prognostic value of pathologic complete response of ipsilateral supraclavicular lymph nodes in breast cancer after neoadjuvant chemotherapy. Ann Transl Med 2019;7:666.

13. Henry BM, Graves MJ, Pękala JR, et al. Origin, branching, and communications of the intercostobrachial nerve: a meta-analysis with implications for mastectomy and axillary lymph node dissection in breast cancer. Cureus 2017;9:e1101.

(English Language Editor: B. Draper)

Cite this article as: Zhang M, Yang L, Hou L, Wang Z, Zhang J. Modified radical mastectomy for level III axillary lymph node clearance: a case report. Gland Surg 2021;10(9):28802884. doi: $10.21037 / \mathrm{gs}-21-567$ 